\title{
Educación ambiental formativa universitaria Caso: Vertidos contaminantes utilizados como material de construcción
}

\author{
Colón Gilberto Martínez RehPani* \\ Ecuador \\ María Caridad Valdés Rodríguez ${ }^{* *}$ \\ Cuba \\ Xavier Oswaldo Orellana LeÓN*** \\ Ecuador
}

\section{Introducción}

Dentro del desarrollo de la cultura ambiental universitaria es posible contribuir con innovaciones donde participen los estudiantes universitarios a través de la investigación continua, de bajo costo y al mismo tiempo fomentar cambios tecnológicos relacionados con el medio ambiente y su entorno social, animando a los futuros profesionales a la apertura de nuevos conocimientos, así como de otros materiales de construcción.

Se puede contribuir con la mejora ambiental y el progreso social incorporando educativamente a los jóvenes al desarrollo de competencias laborales actualizadas, y a hacer uso de los conocimientos y

* Doctorando en Ciencias de la Educación. Universidad Católica Santiago de Guayaquil. Contacto: gilberto.martinezr@hotmail.com

* Doctora en Ciencias Pedagógicas. Universidad de las Ciencias Informáticas. Contacto: mvaldes@uci.cu

**Ing. Civil. Universidad Católica Santiago de Guayaquil. Contacto: xaore91@ gmail.com 
habilidades de la población local, junto con la revalorización de la educación universitaria institucional. A modo de ejemplo se presenta un proceso metodológico de bajo costo, desarrollado por estudiantes universitarios, que hace posible demostrar que sí es factible utilizar los lodos procedentes de las plantas de tratamiento de aguas residuales, instaladas en la ciudad de Guayaquil, en la elaboración de ladrillos cerámicos.

En el presente trabajo se ilustra también que es posible minimizar los impactos ambientales y sociales que se pudieran producir por la disposición final de estos fangos y lodos residuales, y conseguir utilizar este residuo peligroso como material de construcción. Este proyecto propone un nuevo uso de los lodos de aguas residuales que actualmente se depositan en el relleno sanitario de Las Iguanas de la ciudad de Guayaquil.

Simultáneamente, se está tratando de potenciar competencias profesionales, de forma gradual, en todas las carreras universitarias, a fin de lograr resultados favorables en la integración de tecnologías, la ciencia y el pensamiento innovador de la educación, en favor del desarrollo humano sostenible y la Responsabilidad Social Universitaria (RSU).

\section{Antecedentes}

La educación ambiental es un proceso real cuando hablamos de la prosperidad colectiva, y se fundamenta en la necesidad de mejorar, proteger y conservar el medio ambiente, base del sustento de la sociedad humana. Debe ser un proceso educativo permanente, dirigido a estimular la necesidad de universalizar la ética humana e inducir a las personas a adoptar actitudes y comportamientos consecuentes, que aseguren la protección del medio ambiente y el mejoramiento de la calidad de vida de la humanidad.

El medio ambiente se considera como un sistema particularmente complejo y continuo, donde se manifiestan interrelaciones dinámicas entre lo biótico, abiótico, socio cultural, económico, histórico y construido. En él se centra la visión de un planeta interconectado que contempla la propia existencia del hombre, su cultura y sus relaciones 
sociales, por lo que el cuidado de la naturaleza debe constituir uno de los objetivos a ser priorizados por todos los sistemas educativos existentes en el mundo.

La ciencia, la tecnología y la innovación aportan una amplia plataforma teórica y metodológica, para que la educación ambiental constituya una fuente educativa, muy necesaria para el desarrollo en los educandos, de una cultura a favor de la protección del medioambiente con responsabilidad social, pero se requiere de un diseño de acciones formativas para aprovechar estos conocimientos con eficiencia y eficacia.

La consideración hacia el medio ambiente no se desarrolla por sí sola sin la influencia orientadora del educador, la familia y la sociedad en general. Muchos eventos y episodios son la expresión de una evolución creciente de la sensibilidad y conciencia humanas, acerca de la gravedad de los problemas ambientales y de la necesidad de la educación para controlarlos. En la Cumbre de la Tierra, celebrada en Río de Janeiro en 1992, se reconoció la indisoluble vinculación del desarrollo económico con el medio ambiente y se proclamó la dignidad de la persona humana.

La Unesco ha declarado, en el "Decenio de las Naciones Unidas para el Desarrollo Sostenible" (2005-2014), que el principio fundamental reside en el uso de la educación formal como un camino práctico hacia el cambio de valores, actitudes y modos de vida, para que se pueda lograr alcanzar un futuro sostenible y la evolución hacia sociedades más justas.

En muchos países existen leyes bien definidas a favor del medio ambiente, pero está claro que la solución a estos problemas no radica solo en la existencia de una legislación apropiada sino que en cada lugar del planeta es necesario también buscar enfoques, vías, métodos y procedimientos que favorezcan la aplicación práctica, efectiva y consecuente de acciones para la protección del medio ambiente. Las instituciones educativas pueden contribuir de manera determinante a través de la formación con responsabilidad social de sus recursos humanos.

En el marco del respeto al medioambiente, la naturaleza, la vida, las culturas y la soberanía, en Ecuador y en otros países, el Sistema Nacional de Ciencia, Tecnología, Innovación y Saberes Ancestrales, tiene 
entre sus finalidades el desarrollo de tecnologías e innovaciones que mejoren la calidad de vida y contribuyan a la realización del buen vivir, impulsen la producción nacional y eleven la eficiencia y productividad.

Sin embargo, existen contradicciones entre la educación que se requiere poseer desde las instituciones educacionales, sociales y familiares y el desempeño de la actividad humana por la preservación y cuidado en los ambientes naturales ya que, 1) las actividades humanas afectan a los ambientes naturales, reduciéndolos en su extensión y haciendo que desaparezcan especies nativas de animales y plantas, y 2) los ambientes naturales son el soporte de donde se extraen recursos indispensables para el hombre, algunos de los cuales corren el riesgo de terminarse.

\section{La responsabilidad social y las instituciones educativas}

El análisis de algunos fundamentos llevó a los autores a reflexionar en la necesidad del diseño y la implementación futura de una estrategia formativa de enseñanza que, desde este trabajo investigativo, encuentre en la responsabilidad social de la universidad, una de las bases para la generación de nuevos conocimientos que contribuyan a modificar el mundo en el que se vive y su conservación, para mejorar la calidad de vida en el planeta Tierra, en el presente y futuro de las generaciones.

A partir de la segunda mitad del siglo xx han aumentado las preocupaciones por los problemas ambientales, y es cuando se vuelve a repensar en el medio ambiente, debido a los problemas de contaminación, extinción de especies nativas, degradación de bosques, incremento de la pobreza, entre otros. De una manera u otra los problemas ambientales están afectando negativamente la calidad de vida de las poblaciones humanas. La protección del medio ambiente se ha convertido en una necesidad de primer orden para garantizar el desarrollo económico y social y, sobre todo, para la salud y supervivencia de la especie humana.

El principio de pertinencia, consagrado en algunas Constituciones, consiste en que la Educación Superior responda a: 1) las expectativas y necesidades de la comunidad, 2) la planificación nacional y al régimen 
de desarrollo, 3) el desarrollo científico, humanístico y tecnológico y, 4) la diversidad cultural.

Las actividades de formación y de investigación deben de estar acompañadas de una reflexión sobre las consecuencias sociales de esos procesos. Esa es la dimensión moral que diferencia cultura y ciencia. Es necesario preparar estudiantes para su encuentro con problemas reales y sus responsabilidades sociales, para el análisis crítico, la reducción de la brecha entre ricos y pobres y la reducción de la exclusión social.

La responsabilidad social universitaria es la que propone la investigación con innovación ambiental urgente y vinculada a las necesidades mismas de la comunidad, cuidando que este eje principal de responsabilidad social, sea pertinente y práctico, de impacto en el mejoramiento de las condiciones de vida de las personas y muy alejado de la simple oratoria.

La responsabilidad social universitaria no es una etiqueta o una moda temporal, sino asumir valores sociales y compromisos con la justicia social y el desarrollo responsable. Es la puesta en práctica de los postulados de la Misión o del Plan Estratégico de Desarrollo Institucional (Pedi) de la Universidad. Se inicia con la investigación desarrollada en los salones de clase como un acto de servicio a la sociedad y es coherente con el principio universal de servicio al prójimo.

La contaminación actual de la atmósfera, cobertura vegetal y cuerpos de agua como ríos, lagos y mar, son indicativos de lo poco que se ha avanzado en las problemáticas ambientales, lo que hace necesario, entre otras tareas, promover actividades universitarias a nivel de los futuros profesionales que permitan proveerlos de las referencias y concientización fundamental para cuidar y conservar su entorno.

En la ciudad de Guayaquil, las migraciones han impactado a una comunidad golpeada por el desempleo, y ahora se ha instaurado un serio desafío de convivencia entre personas de amplia diversidad cultural. En la presente investigación se plantea una integración desde el enfoque ambiental, educativo y espontáneo de los nuevos profesionales, buscando la proximidad entre migrantes y la sociedad de acogida, que reconoce la situación de cada comunidad para la conformación de una igualdad compartida. 
Fueron de mucha importancia las entrevistas con actores de distintos ámbitos sociales y profesionales oponentes significativos, cuyas investigaciones y quehacer docente se relacionan con la presente temática, o que ocupan cargos de decisión a nivel de empresa pública y/o privada, que influyeron en las acciones específicas relacionadas, así como también, profesionales de los medios de comunicación que contribuyeron con la difusión de opiniones, concurso estudiantil de innovaciones, reflexiones y noticias relacionadas entregadas a la ciudadanía.

\section{Proceso metodológico de bajo costo}

En el Noroeste de Guayaquil, uno de los asentamientos informales llamado La Ladrillera, ubicado en el Bloque 9, Flor de Bastión, a la altura del km 14,5 de la vía a Daule, sobrevive precariamente desde hace 23 años, cuando se inició su proceso migratorio de familias procedentes generalmente de comunidades rurales de Manabí, Guayas, Esmeraldas y Los Ríos. Sus viviendas aún son básicas, en su mayoría de caña, plástico y cartón, techos de zinc, piso de tierra o tablas.

Muchas de estas familias son numerosas y entre las actividades que esta comunidad realiza está la de hacer ladrillos artesanales de arcilla cocida, utilizando una técnica antigua y existente en muchas localidades a nivel mundial. De manera experimental se intentó que esta comunidad, conjuntamente con estudiantes de la Facultad de Ingeniería de la Universidad Católica de Santiago de Guayaquil, pueda fabricar materiales de construcción distintos de los que ya realiza y mejorar así sus prácticas actuales, de modo que se puedan ampliar sus posibilidades de mercado.

En esta comunidad de trabajo se percibió a una ciudadanía participativa, que permitió razonamientos y experiencias con los universitarios en los procesos de aprendizaje, y el inicio de una propuesta formativa futura en este entorno socioeducativo, con sentido compartido entre estudiantes que están por graduarse y un grupo de personas de la mencionada comunidad, entusiastas en la observación, comparación, investigación y síntesis. 
Para lograr la fabricación de ladrillos utilizando parcialmente los lodos provenientes de aguas residuales, se efectuaron visitas a distintas Plantas de Tratamiento de Aguas Residuales (PTAR) ubicadas en la ciudad de Guayaquil. Se analizó el manejo logístico de estos lodos y también el funcionamiento de las plantas de tratamiento. Se estudiaron los volúmenes de lodos que se producen y se localizó el sitio de muestreo apropiado para el presente proyecto experimental.

También fue necesario analizar el proceso de vertido que se realiza en el relleno sanitario de Las Iguanas, donde se depositan parte de estos lodos sobrantes, provenientes de algunas plantas de tratamiento de aguas residuales y también de canales de drenaje de aguas servidas de la ciudad de Guayaquil.

El sitio de muestreo seleccionado por estudiantes de la Facultad de Ingeniería, para la obtención de material de ensayo para la fabricación experimental de ladrillos artesanales con adiciones de lodos residuales fue una planta, cuyo proceso de deshidratación se realiza de forma natural, aprovechando los rayos uv en una cámara construida para el efecto. Estos lodos estaban biológicamente estabilizados y podían ser utilizados como un componente en la fabricación de ladrillos artesanales con adiciones de lodos residuales.

En la elaboración de ladrillos artesanales experimentales con adiciones de lodos residuales y la posterior quema de los mismos, se involucró a la comunidad de La Ladrillera, cuyos operarios son trabajadores hábiles en esta actividad artesanal. Se aseguró así la calidad de las muestras que se iban a fabricar. Esta comunidad está asentada de manera informal en el Noroeste de la ciudad de Guayaquil y su actividad es fabricar ladrillos con las arcillas del mismo sitio donde se han asentado. Se entrenó a los voluntarios de La Ladrillera y se les proporcionó equipos de protección individual por la presencia de patógenos en las muestras.

La zona donde está asentada la comunidad posee topografía ondulada, donde las depresiones se han rellenado geológicamente con sedimentos arcillosos, erosionados de las laderas de las elevaciones constituidas por arcillas residuales de la formación Piñón de la ciudad de Guayaquil. Estas arcillas sedimentarias de las depresiones mencionadas constituyeron la matriz principal del ladrillo artesanal con adiciones 
de lodos residuales que se fabricó. A esta arcilla sedimentaria natural del sitio se le adicionaron los porcentajes previstos de lodos residuales, muestreados en la planta de tratamiento.

Las adiciones porcentuales experimentales escogidas fueron cinco: 1) ladrillos $A$ ( $0 \%$ de adición de lodos residuales), 2) ladrillos $B(10 \%$ de adición de lodos residuales), 3) ladrillos C (20\% de adición de lodos residuales), 4) ladrillos $\mathrm{D}$ (30\% de adición de lodos residuales), y 5) ladrillos E (100\% de adición de lodos residuales).

El proceso de secado de los ladrillos crudos se realizó de manera natural, aprovechando la radiación solar. El secado duró 15 días y durante el acompañamiento técnico del mismo, también participaron de esta actividad ambientalmente educativa, los voluntarios de la comunidad de La Ladrillera, sensibilizados por la afectación que la misma tendrá en su calidad de vida.

Concluido el proceso de secado natural de todas las muestras de ladrillos crudos, la comunidad procedió a armar la pirámide de cocción convencional para el respectivo incremento de la temperatura y el cocimiento de los ladrillos experimentales. Los voluntarios de $\mathrm{La}$ Ladrillera construyeron una pirámide de 5.000 ladrillos aproximadamente y procedieron a realizar la cocción, la misma que, de acuerdo con las investigaciones realizadas por Gnecco, M. y Marquina, J. en el año 2.000 , estos ladrillos debieron superar los $750^{\circ} \mathrm{C}$ de temperatura.

Los ladrillos estuvieron sometidos a esta temperatura aproximadamente durante 3 días. Concluido este proceso se pudo observar que los ladrillos elaborados con el $100 \%$ de lodos residuales sufrieron la quema parcial de la materia sólida. La materia orgánica se quema cuando la temperatura supera los $550^{\circ} \mathrm{C}$ y esta funcionó como combustible.

\section{Resultados}

Concluido el proceso de quema, los especímenes con adiciones de lodos residuales fueron conducidos al laboratorio para ensayos de absorción, densidad y de compresión simple de los ladrillos, en posición de canto, sugestiva de la posición en la que trabajarían en una pared convencional. Además de las mediciones geométricas y de peso convencionales. 
El cuadro 1 muestra los resultados obtenidos en las pruebas de compresión simple en los ladrillos con adiciones de lodos residuales con $0,10,20$, y $30 \%$.

Se puede deducir que los ladrillos de arcillas residuales de la formación Piñón de la ciudad de Guayaquil, con adiciones de hasta un $20 \%$ de lodos residuales provenientes de las plantas de tratamiento no presentan diferencias importantes en su resistencia a la compresión simple en relación con los mismos ladrillos sin adiciones de estos lodos residuales.

Los ladrillos con adiciones del $10 \%$ de lodos residuales presentaron mayores resistencias que los ladrillos sin adiciones, presumiblemente por el llenado de espacios vacíos intersticiales en la mezcla total por la parte mineral inorgánica contenida en los lodos residuales.

Cuadro 1. Distribución de la resistencia a la compresión simple promedio obtenida en las muestras ensayadas. Elaboración propia.

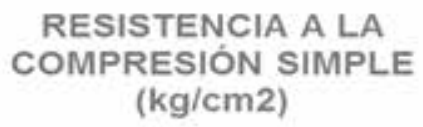

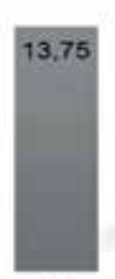

A

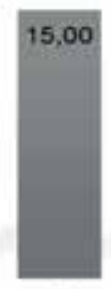

B

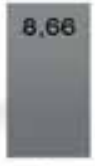

c

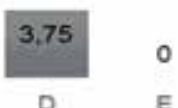

E

Durante la fabricación y quema de ladrillos utilizando el 100\% de lodos residuales se pudo determinar que fue consumido el $77 \%$ en peso de la materia sólida. Se pudo concluir además que el remanente encontrado, posterior a la quema de estos ladrillos, es del $23 \%$ en peso y corresponde a material mineral no orgánico. 


\section{Discusión}

La fabricación lograda de estos ladrillos con adiciones de lodos residuales de las plantas de tratamiento de la ciudad de Guayaquil permitiría abrigar la idea de que se podría implementar a futuro, una planta automática sin participación directa de personas, que logre procesar conjuntamente con las arcillas residuales y sedimentarias de la ciudad de Guayaquil, los lodos residuales y otros productos contaminantes industriales de difícil disposición, así como también los materiales obtenidos en la limpieza de canales de aguas residuales de la ciudad.

De esta manera será posible aprovechar estos subproductos de múltiples maneras en la construcción, tales como ladrillos para paredes de mampostería, ladrillos para relleno de losas aligeradas, paneles para vivienda social, entre otros.

Estos resultados corresponden a los primeros que se realizan para ladrillos artesanales fabricados con arcillas de la formación Piñón del noroeste de Guayaquil, con adiciones de lodos residuales. El presente trabajo es perfectible y esta investigación actualmente continua realizándose en su siguiente etapa, buscando fabricar materiales de construcción de uso masivo para un posible uso en divisiones para vivienda social, utilizando los lodos residuales obtenidos de cámaras sépticas, lagunas de oxidación y de la limpieza de canales de drenaje de aguas residuales de la ciudad de Guayaquil, considerados productos contaminantes peligrosos.

\section{Conclusiones}

El presente estudio contribuye a incrementar el compromiso de las Universidades con sus próximos profesionales, de mejorar el apoyo que estas prestan en la asesoría, formación y/o perfeccionamiento de microempresas y actividades productivas tecnológicas locales que necesitan de investigación aplicada para desarrollar o ampliar emprendimientos ambientales, con escasos recursos económicos.

Las iniciativas que se están desarrollando lentamente en la Universidad Católica de Santiago de Guayaquil poseen un capítulo de 
sensibilización y concientización ambiental que nace con la educación, y va a contribuir con soluciones reales que no solo se transformarán en infraestructuras, sino que harán sentido en la mente de los profesionales bajo principios de sostenibilidad, para fomentar el desarrollo económico y social de las comunidades donde actúen.

La responsabilidad social universitaria trasciende lo empresarial, cuida el medio ambiente y se preocupa por las personas humanas. Los moradores pobres de La Ladrillera de Guayaquil, de alguna manera están participando de la Educación Superior y reciben formación en consonancia con su circunstancia, para la mejoría en los procesos de fabricación de sus productos y nuevas alternativas para el mercado.

Para pensar en un futuro desarrollo rural sostenible, en este momento se tornaría muy necesario educar a los jóvenes profesionales que son cercanos actores sociales de sus diversos entornos, reforzándoles importantes valores ambientales e integrándolos con las diversas comunidades, de tal manera que ellos puedan fomentar en su nuevo accionar profesional, actitudes significativas para la conservación de las distintas zonas del país, siendo capaces también de entrenar a los pobladores para que se puedan mitigar los impactos ambientales que se generen en su propio contexto, de manera personal y/o colectiva.

Para lograrlo, la Universidad debe responder entonces a las urgentes exigencias de transformación de una sociedad, a través de la docencia, la investigación y su vinculación con la sociedad, consagradas en la Carta Magna, respaldando la justicia y equidad social. Son los desafíos actuales del desarrollo humano sustentable que hacen parte de los anhelos de la comunidad universitaria para lograr acercarse a los Objetivos de Desarrollo Sostenible en América Latina. 


\section{Referencias}

Albornoz, M., et. al. (2012). Ciencia, tecnología e innovación para el desarrollo y la cohesión social. Madrid: OEI.

Asamblea Nacional. (2008). Constitución de la República del Ecuador.

Aznar, P., et. al. (2014). La sostenibilidad en la formación universitaria: desafíos y oportunidades. Revista Educación. España XX1, 17.

Bárcena, A., Narcís, E. (2011). Educación, desarrollo y ciudadanía en América Latina. Propuestas para el debate. Santiago de Chile: Cepal.

Citma (2010). Estrategia Nacional de Educación Ambiental 2.010- 2.015. La Habana: Citma/Cigea.

Domínguez, J. (2012). Conceptualización sobre la responsabilidad social específica de una universidad católica, en Domínguez, J., Rama C. (Eds.), La responsabilidad social Universitaria en la Educación a Distancia. (pp. 53-74). Chimbote, Perú: Uladech Católica. http://repositorio.uladech.edu. pe/bitstream/handle/ULADECH_CATOLICA/92/LA_RESPONSABILIDAD_SOCIAL_EN_LA_EDUCACION_A_DISTANCIA.pdf

Valdiviezo, C., Salcedo, K., Sánchez, L. (2015). Transferencia del conocimiento y de las prácticas de RSU contribuyen a reducir la pobreza extrema, en Domínguez, J., et al., Responsabilidad Social de las Organizaciones (RSO): Avances y propuestas en América Latina. (pp. 367-388). Trujillo, Perú: Uladech Católica. http://repositorio.uladech.edu.pe/bitstream/handle/ULADECH_CATOLICA/83/SIRSO.pdf)

Dondi, M. M. (1997). Recycling of industrial and urban wastes in brick production. A review. Part 2. Vol. 13. Tile \& Brick International.

Escobar, R. A. (2012). La Doctrina Social de la Iglesia: Fuentes, Principios y Concepción de los Derechos Humanos. Revista Prolegómenos. Derechos $y$ Valores. 15, 30, 99-117.

Ezquerra, G., Gil, J. (2014). De la obligación institucional a la conciencia ambiental: El reto de la ambientalización en la Universidad de La Habana en Revista Estudios del Desarrollo Social: Cuba y América Latina. Flacso/Cuba.

Francisco, P. (2015). Carta Encíclica Laudato Si: Sobre el cuidado de la casa común. Vaticano: Tipografía Vaticana.

Gnecco, M., \& Marquina, J. (2000). Ladrillos que ahorran energía: manual para pequeños productores de ladrillos. Lima. 
Martínez, G., (2015). Memorias III Congreso Internacional. Tecnología, Universidad y Sociedad (TUS). La Educación Ambiental: actividad transversal urgente en la formación de los estudiantes universitarios. (pp. 275-283). Guayaquil. Recuperado en http://ecotec.edu.ec/congresotus/ memorias-tus.pdf

Martínez, G., Orellana, X. (2015). Fabricación de ladrillos con lodos y fangos residuales depositados en el relleno sanitario "Las Iguanas", provenientes de las plantas de tratamiento de aguas servidas de Guayaquil y canales de drenaje, en Odebrecht Ingeniería \& Construcción International (eds.), Premio Odebrecht para el Desarrollo Sostenible. Los diez mejores proyectos. (pp. 22-39). Quito. Recuperado en http://www.premioodebrecht.com.ec/wp-content/uploads/2016/08/Libro-premio-Odebrecht-2015-low1.pdf

Montuschi, L. (2007). El bien común, la responsabilidad social empresaria y el pensamiento social de la iglesia. Buenos Aires: Universidad del CEMA.

ONU. (1992). Conferencia de las Naciones Unidas sobre el Medio Ambiente y el Desarrollo. Cumbre de la Tierra. Río de Janeiro: Naciones Unidas.

ONU. (2010). Programa de las Naciones Unidas para el Desarrollo (PNUD). Informe.

Organización de Estados Iberoamericanos oer. (2012). Ciencia, Tecnología e Innovación para el desarrollo y la cohesión social. Madrid: OEI.

Organización Internacional de Normalización Iso (2010). Norma Iso26.000:2010. Guía de Responsabilidad Social.

Slim, J., \& Wakefield, R. (1991). The utilization of sewage sludge in the manufacture of clay bricks. Vol. 17. Water SA.

Tay, J. H. (1987). Bricks manufactured from sludge. Journal of Environmental Engineering.

Unesco (2005). Decenio de las Naciones Unidas de la Educación para el Desarrollo Sostenible 2.005-2.014. París: Talleres Unesco.

Unesco (2012). Educación para el Desarrollo Sostenible. Libro de Consulta. Instrumentos de aprendizaje y formación. No. 4. Paris.

Unesco (2015). Foro Mundial sobre la Educación. Declaración de Incheon. Educación 2.030.

Villarroel, J. (2011). II Congreso Nacional de Vinculación de la Educación Superior con la Colectividad. Vinculación de la educación superior: ¿Servir al mercado o cumplir con la Responsabilidad Social? 